\title{
Modelling of Dual-Junction Solar Cells including Tunnel Junction
}

\author{
Abdelaziz Amine, Yamina Mir, and Mimoun Zazoui \\ Laboratory of Condensed Matter and renewable energy, University Hassan II Mohammedia-Casablanca, \\ Faculty of Sciences and Techniques, BP 146, Boulevard Hassan II, Mohammedia 28800, Morocco \\ Correspondence should be addressed to Mimoun Zazoui; zazouimimoun@yahoo.fr
}

Received 8 May 2013; Revised 5 August 2013; Accepted 15 August 2013

Academic Editor: Xia Xiang

Copyright (C) 2013 Abdelaziz Amine et al. This is an open access article distributed under the Creative Commons Attribution License, which permits unrestricted use, distribution, and reproduction in any medium, provided the original work is properly cited.

\begin{abstract}
Monolithically stacked multijunction solar cells based on III-V semiconductors materials are the state-of-art of approach for high efficiency photovoltaic energy conversion, in particular for space applications. The individual subcells of the multi-junction structure are interconnected via tunnel diodes which must be optically transparent and connect the component cells with a minimum electrical resistance. The quality of these diodes determines the output performance of the solar cell. The purpose of this work is to contribute to the investigation of the tunnel electrical resistance of such a multi-junction cell through the analysis of the current-voltage $(J-V)$ characteristics under illumination. Our approach is based on an equivalent circuit model of a diode for each subcell. We examine the effect of tunnel resistance on the performance of a multi-junction cell using minimization of the least squares technique.
\end{abstract}

\section{Introduction}

Currently, multijunction III-V solar cells have the highest solar cell energy conversion efficiencies $(40 \%)[1,2]$, and they are still under investigation for even higher values. Stacked $p-n$ junctions with different band gap energies, such as dual junctions GaInP/GaAs, can pave the way for the solar spectrum efficiently. In a multijunction solar cell, the subcells are interconnected through tunnel junctions $[3,4]$, in order to align Fermi levels. These tunnel junctions must have a low electrical resistivity [5-8], a high optical transmission, and a high peak tunnelling current density $[4,9,10]$.

Due to the complexity of the electrical and optical interaction between different layers [11, 12], pure experimental optimization of these structures is expensive and time consuming. A suitable simulation is desirable to help accelerating the experimental optimization. During simulation, different physical processes beyond the standard drift equations of electrical transport have to be considered as follows: existence of hetero-interfaces and interference effects. These processes have been partly implemented in most of simulation programs. There exist commercial semiconductor simulation programs which give excellent results for modelling single-junction solar cell $[3,8]$. However, taking into consideration the mentioned physical process, a correction of description for the series interconnection of individual subcells must be included $[5,6,13]$.

The current-voltage $(J-V)$ characteristic under illumination is the most important parameter of any solar cell $[5,7,14]$. This characteristic allows determining the parameters of the cell and thus predicting its performances. This is done by fitting the illumination $(J-V)$ curve into a model containing the parameters.

There are two basic models to describe the $J-V$ characteristics of a solar cell: a simple one, which considers the solar cell as a single p-n junction diode with parasitic resistances, and a more elaborated model which takes into account the recombination centres present in the solar cell junction.

There are several methods to extract the solar cell parameters. A relatively simple one exists to estimate the series and shunt resistances of the cell using the slope of the currentvoltage $(J-V)$ curve in conditions of open-circuit voltage and short-circuit current, respectively. Other methods use complicated numerical analysis based on Lambert's W function, calculating the resistance from the difference between the diode characteristics in dark and under illumination 
[15]. These methods necessitate accurate extraction of many parameters from a nonlinear equation. In most cases, the ideality factor is assumed to be equal to 1 (i.e., the parallel resistance is neglected) so that all parameters cannot be properly extracted. Moreover, in most studies, the $(J-V)$ characteristics obtained from the extracted parameters are not compared with the measured $(J-V)$ ones, making it difficult to evaluate the accuracy of the methods.

In this work, we present a method to extract the parameters of a dual junction solar cell, that is, including a tunnel junction. It uses the one diode model [16]. Taking into consideration current matching between subcells, we derive the analytical expression of the $J-V$ curve using Lambert's W function. As approximation, we neglect the series resistance of each subcell, considering only the tunnel resistance $r_{\text {tun }}$.

The objective of this work is to compare the $J-V$ characteristics derived from these parameters with experiment data available in the literature [7] in order to test the validity of our procedure. Then, we apply our method to extract different parameters of dualjunction such as the tunnel resistance of a particular cell.

\section{Modelling a $J-V$ Dual Junction}

Introduction of tunnel junctions between subcells is the way of monolithically building a multijunction solar cell. These tunnel junctions must cause minimum losses of the conversion efficiency. This implies that they exhibit a low series resistance and a minimum optical absorption. A tunnel junction diode is a diode made of a wide band gap, highly doped material. This reduces the length of the depletion region so that electrons can easily tunnel through (the tunnel effect) [17].

In order to identify the relationship between the quality of a tunnel junction and the semiconductor parameters used to design its structure, necessary simplifications will be used. We employ the one exponential model, taking only the recombination current term into account. The tunnel junction will be considered ideal, meaning that it will always work in the ohmic region and its behaviour can be modelled as a voltage drop which depends linearly on the current flowing through it [18].

To calculate the $J-V$ curve of GaInP/GaAs dual junction including AlGaAs tunnel junction, we start from the expressions for the voltages at each junction for a given current. In this case, both subcells in the dual junction solar cell are connected in a series configuration, and the device is accessed through two terminals, as shown schematically in Figure 1. The voltage at the whole dual-junction solar cell is given by

$$
V_{\text {dual }}=V_{\text {top }}+V_{\text {tun }}+V_{\text {bot }} \text {, }
$$

where $V_{\text {top }}, V_{\text {bot }}$, and $V_{\text {tun }}$ are the voltages at GaInP top cell GaAs bottom cell, and AlGaAs tunnel junction respectively.

The currents flowing through the two subcells as well as the tunnel diode are assumed to be current matched [10]; that is, the photogenerated current in both subcells is considered to be of equal value. It is determined by the subcell, delivering the lowest current, again that

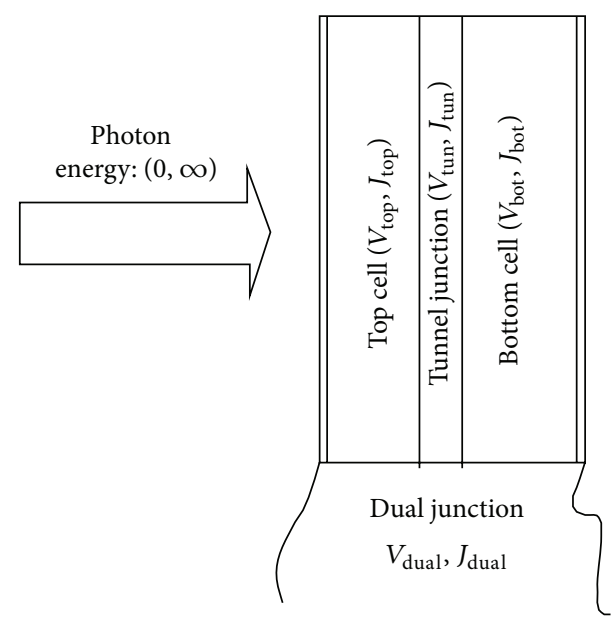

Figure 1: Scheme of the two terminals, of dual-junction solar cell.

the importance of the current matching is evident, the maximum efficiency $(\eta)$ and the fill factor (FF) - that is, cells with a high fill factor, have a low equivalent series resistance and a high equivalent shunt resistance, and consequently the series and shunt resistances of each subcell top and bottom are neglected $[5,8,9]$. With these conditions, an analytical expression for the illumination $J-V$ characteristic of the dual-junction solar cell can be developed. The general one-exponential model for each subcell [9] which is going to be used is as follows:

$$
J=J_{L}-J_{0} \exp \left(\frac{V-r_{s} J}{V_{t}}\right) \quad \text { where } V_{t}=\frac{k_{B} T}{q},
$$

where $J_{0}, J_{L}$ are saturation current density and photogenerated current density, respectively, $r_{s}$ is series resistance, $q$ is the charge, $V$ is the bias voltage, $k_{B}$ is the Botzmann constant, and $T$ is the temperature. follows

The voltages at each subcell and tunnel junction are as

$$
\begin{gathered}
V_{\text {top }}=r_{\text {top }} \cdot J_{\text {top }}+V_{t} \ln \left(\frac{J_{L \text { top }}-J_{\text {top }}}{J_{0 \text { top }}}\right), \\
V_{\text {bot }}=r_{\text {bot }} \cdot J_{\text {bot }}+V_{t} \ln \left(\frac{J_{L \text { bot }}-J_{\text {bot }}}{J_{0 \text { bot }}}\right), \\
V_{\text {tun }}=r_{\text {tun }} \cdot J_{\text {tun }} .
\end{gathered}
$$

Since the current density through both subcells must be of equal value and assuming that the subcells are current matched at $T=300 \mathrm{~K}$,

$$
J_{\text {top }}=J_{\text {bot }}=J_{\text {tun }}=J_{\text {dual }} \text {. }
$$

We neglect the series resistance of each subcell and consider only the tunnel resistance $r_{\text {tun }}$.

Hence, the expression of the current density of dual junction is

$$
J_{\text {dual }}=J_{L}-\sqrt{J_{0 \text { top }} \cdot J_{0 \text { bot }}} \exp \left(\frac{V_{\text {dual }}-r_{\text {tun }} J_{\text {dual }}}{2 V_{t}}\right) .
$$


This expression is similar to the general form of the single junction solar cell one exponential model, but using the geometric average of the recombination currents as the exponential prefactor and introducing a factor 2 in the denominator of the exponential term.

Accordingly, the open circuit voltage is

$$
\begin{gathered}
V_{\mathrm{OC}}=2 V_{t} \ln \left(\frac{J_{L}}{\sqrt{J_{0 \text { top }} \cdot J_{0 \text { bot }}}}\right), \\
V_{\mathrm{OC}}=V_{\text {OC top }}+V_{\text {OC bot }}
\end{gathered}
$$

where $V_{\mathrm{OC} \text { top }}$ and $V_{\mathrm{OC} \text { bot }}$ are the open circuit voltages of each subcell top and bottom, respectively.

And short circuit current can be derived as follows:

$$
J_{\text {sc }}=J_{L}-\sqrt{J_{0 \text { top }} \cdot J_{0 \text { bot }}} \exp \left(\frac{J_{\text {sc }} \cdot r_{\text {tun }}}{2 V_{t}}\right) .
$$

From (5), the current $J_{\text {dual }}$ can be derived as follows:

$$
\begin{aligned}
J_{\text {dual }}= & \left(r_{\text {tun }} J_{L}+2 V_{t} \cdot \text { Lambert } \mathrm{W}\right. \\
& \left.\times\left(-\frac{\sqrt{J_{0 \text { top }} J_{0 \text { bot }}} \cdot r_{\text {tun }}}{2 V_{t}} \exp \left(-\frac{-V_{\text {dual }}+r_{\text {tun }} J_{L}}{2 V_{t}}\right)\right)\right) \\
& \times\left(r_{\text {tun }}\right)^{-1} .
\end{aligned}
$$

The purpose of algorithm is to fit a nonlinear characteristic $J-V(8)$ which suits the given data appropriately and subscribes to the minimization of the sum of the squared differences between the actual data and the predicted one. The error $\varepsilon$ thus is defined as

$$
\varepsilon=\sum_{i=1}^{N}\left(J_{i}-J\left(V_{i}, p\right)\right)^{2}
$$

where $N$ is the number of measured $J(V)$ pairs denoted by $\left(V_{i}, J_{i}\right) ; J(V, p)$ is the theoretical current for voltage $V$ as predicted by a model containing several parameters, represented by $p$, that are the variables used to minimize the error.

Being put into effect with Matlab Software, we have shown the experimental data and simulation $J-V$ characteristic under illumination of the dual-junction solar cell using the one-diode model program to extract the important features parameters of each subcell (top and bottom) such as recombination current $J_{0 \text { top }}, J_{0 \text { bot }}$ and resistance tunnel $r_{\text {tun }}$ (so as to give more information about the electrical performance dual junction) restricted solely in domain boundaries definition. For so doing, we have chosen the minimum and maximum limit: $L_{b}<p<U_{b}$ with $p=\left(J_{0 \text { top }}, J_{0 \text { bot }}, r_{\text {tun }}\right)$.

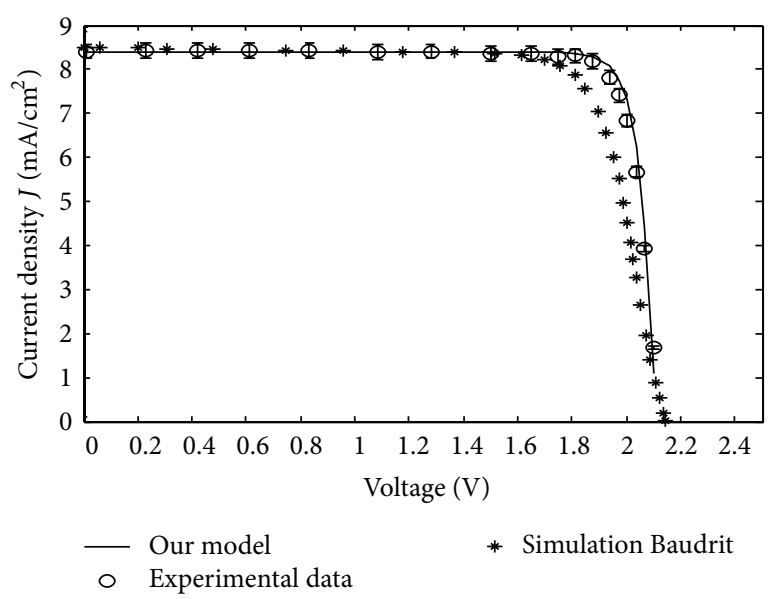

FIGURE 2: The experimental and simulation data for dual junction current density versus voltage. Circles correspond to experimental data, stars to results of Baudrit and Algora [7] and line to our model.

\section{Analysis of Experimental Data}

We applied our model to results given by Baudrit and Algora [7]. The sample consists of GaInP/GaAs dual solar cell in presence of a p ${ }^{++} \mathrm{GaAs}-\mathrm{n}^{++} \mathrm{GaAs}$ tunnel junction. Parameters of the one-diode model were extracted using a program that employed the iterative method. Matlab was used to create a program that made use of a user-defined parameter initialization scheme to set the boundaries of the iteration and minimize computing time. The algorithm used to extract the parameters such as the resistance tunnel $r_{\text {tun }}$ of the one-diode model utilized both approximations and iterative techniques.

In Figure 2, we show the measured and simulated $J-V$ characteristic under illumination of the dual solar cell using the one-diode model parameter extraction program. We can see clearly that the curve from our model fit very well the experimental data in comparison with the simulation [7]. Consequently, simulation is not taken into consideration the resistance tunnel $r_{\text {tun }}$.

In Table 1, we give the values of the characteristics of the dual junction compared with experience and simulation. The parameters $J_{\max }$ and $V_{\max }$ are the maximum current density and voltage, respectively.

The obtained parameters are in good accordance with the growth condition and technological process data $[7,12,17]$. Unlike precedent research conducted on this ground [7], we have come up with method for extracting the resistance tunnel $r_{\text {tun }}$, the recombination current density $J_{0 \text { top }}$ and $J_{0 \text { bot }}$.

In order to validate our model, we analyse dual-junction solar cell including tunnel junction, the sample consists of $\mathrm{GaInP} / \mathrm{GaAs}$ on Ge substrate grown by MOCVD, which is $\mathrm{n}^{+}$(emitter) on $\mathrm{p}$ (base) structures. The AlInP windows are n-type doped at level of $7 \times 10^{18} \mathrm{~cm}^{-3}$. The GaAs and GaInP emitters and bases, respectively, doped at $1 \times 10^{18} \mathrm{~cm}^{-3}$ and $3 \times 10^{17} \mathrm{~cm}^{-3}$. The dopants are Si(n) and $\mathrm{C}(\mathrm{n})$ in $\mathrm{AlGaAs}$ tunnel junction and $\mathrm{Si}(\mathrm{n})$ and $\mathrm{Zn}(\mathrm{p})$ in GaAs and GaInP. The GaInP and GaAs are, respectively, $500 \mathrm{~nm}$ and 3,15 $\mu \mathrm{m}$ thick. 


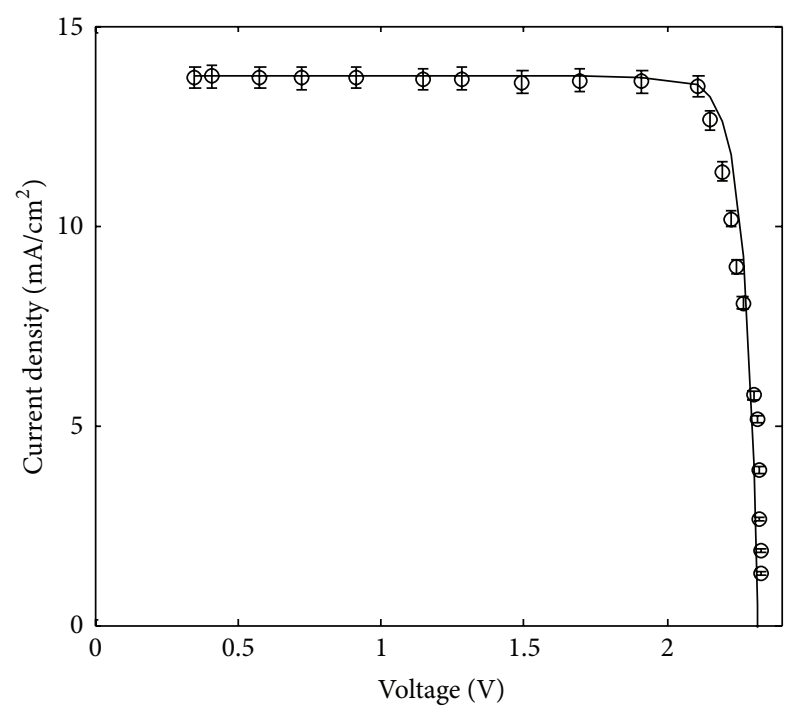

FIgURE 3: Experimental data (circle) and simulated (continuous line) for dual junction GaInP/GaAs.

TABLE 1: Parameters extracted from the experience and simulation by Baudrit and Algora [7] and comparison with our extracted parameters.

\begin{tabular}{lccc}
\hline Parameters & Experience [7] & $\begin{array}{c}\text { Baudrit and } \\
\text { Algora [7] }\end{array}$ & Our model \\
\hline$J_{\text {sc }}\left(\mathrm{mA} \cdot \mathrm{cm}^{-2}\right)$ & 8.4 & 8.4 & 8.4 \\
$V_{\text {oc }}(\mathrm{V})$ & 2.12 & 2.12 & 2.12 \\
$J_{\max }\left(\mathrm{mA} \cdot \mathrm{cm}^{-2}\right)$ & 7.9 & 7.66 & 8.06 \\
$V_{\max }(\mathrm{V})$ & 1.9 & 1.82 & 1.94 \\
FF $(\%)$ & 84 & 78 & 87.8 \\
$\eta$ & 15 & 13.9 & 15.63 \\
$J_{0 \text { top }}\left(\mathrm{mA} \cdot \mathrm{cm}^{-2}\right)$ & - & - & $6.72 \times 10^{-21}$ \\
$J_{0 \text { bot }}\left(\mathrm{mA} \cdot \mathrm{cm}^{-2}\right)$ & - & - & $4.72 \times 10^{-14}$ \\
$r_{\text {tun }}\left(\Omega \cdot \mathrm{cm}^{2}\right)$ & - & - & $4.9 \times 10^{-4}$ \\
\hline
\end{tabular}

TABLE 2: Parameters extracted from our model and comparison with experience.

\begin{tabular}{lcc}
\hline Parameters & Experience & Our model \\
\hline$J_{\mathrm{sc}}\left(\mathrm{mA} / \mathrm{cm}^{2}\right)$ & 13.73 & 13.73 \\
$V_{\mathrm{oc}}(\mathrm{V})$ & 02.32 & 02.32 \\
$J_{\max }\left(\mathrm{mA} / \mathrm{cm}^{2}\right)$ & 13.49 & 13.50 \\
$V_{\max }(\mathrm{V})$ & 02.11 & 02.12 \\
$\mathrm{FF}(\%)$ & 89.34 & 89.84 \\
$\eta$ & 28.46 & 28.62 \\
$J_{0 \text { top }}\left(\mathrm{mA} / \mathrm{cm}^{2}\right)$ & - & $4.60 \times 10^{-23}$ \\
$J_{0 \text { bot }}\left(\mathrm{mA} / \mathrm{cm}^{2}\right)$ & - & $4.83 \times 10^{-15}$ \\
$r_{\text {tun }}\left(\Omega \cdot \mathrm{cm}^{2}\right)$ & - & $4.48 \times 10^{-4}$ \\
\hline
\end{tabular}

In Figure 3, we show the experimental data and simulation $J-V$ characteristic under illumination of the dualjunction solar cell using the one-diode model program to extract the important features of each subcell. In Table 2, we give the values of the characteristics of the dual junction compared with experience.

In Table 2, resuming the main characteristics of dual junction at 1 Sun, the simulation shows a very good fit with the experimental data on the $J-V$ prediction. Further, we have come up with other extractions of each subcells (top and bottom) precisely $J_{0 \text { top }}$ and $J_{0 \text { top }}$ and $r_{\text {tun }}$ which intersect with other researchers' findings $[7,18]$.

\section{Conclusion}

Based on a modelling of dual-junction solar cells including tunnel junction and $J-V$ characterization of multijunction, solar cells were established and applied to semiconductor junctions and devices. In this work, we have given much importance to extract the parameters which are characteristics of the dual junction, using the simulation through fitting by Matlab software. Measurements have been carried on double junction III-V solar cells (GaInP/GaAs) including the AlGaAs tunnel junction, and results have been exploited to extract the parameters to give more information about the electrical performance of dual junction.

\section{References}

[1] R. R. King, D. C. Law, K. M. Edmondson et al., "40\% efficient metamorphic GaInPGaInAsGe multijunction solar cells," Applied Physics Letters, vol. 90, no. 18, Article ID 183516, 2007.

[2] W. Guter, F. Dimroth, M. Meusel, and A. W. Bett, "Tunnel diodes for III-V multi-junction solar cells," in Proceedings of the 20th European Photovoltaic Solar Energy Conference, pp. 515518, Barcelona, Spain, June 2005.

[3] G. Letay, M. Hermle, and A. W. Bett, "Simulating singlejunction GaAs solar cells including photon recycling," Progress in Photovoltaics, vol. 14, no. 8, pp. 683-696, 2006.

[4] G. A. M. Hurkx, D. B. M. Klaassen, and M. P. G. Knuvers, "A new recombination model for device simulation including tunneling," IEEE Transactions on Electron Devices, vol. 39, no. 2, pp. 331-338, 1992.

[5] T. Takamoto, M. Yamaguchi, and E. Ikeda, "Mechanism of $\mathrm{Zn}$ and Si diffusion from a highly doped tunnel junction for InGaP/GaAs tandem solar cells," Journal of Applied Physics, vol. 85 , no. 3, p. $14816,1999$.

[6] I. Garcia, I. Rey-Stolle, B. Galiana, and C. Algora, "A 32.6\% efficient lattice-matched dual-junction solar cell working at 1000 suns," Applied Physics Letters, vol. 94, no. 5, Article ID 053509, 2009.

[7] M. Baudrit and C. Algora, "Modeling of GaInP/GaAs dualjunction solar cells including tunnel junction," in Proceedings of the 33rd IEEE Photovolatic Specialists Conference (PVSC '08), pp. 1-5, May 2008.

[8] H. Shivaganaik and S. H. Jangamshetti, "Modeling and analysis of multi-junction solar cells," in Proceedings of the International Conference on Emerging Trends in Electrical and Computer Technology (ICETECT '11), pp. 174-179, March 2011.

[9] S. M. Sze, Semiconductor Devices, Physics and Technology, John Wiley \& Sons, New York, NY, USA, 2nd edition, 2001.

[10] M. Hermle, S. P. Philipps, G. Letay, and A. W. Bett, "Numerical simulation of tunnel diodes and multi-junction solar cells," in 
Proceedings of the 33rd IEEE Photovoltaic Specialists Conference, pp. 1-4, San Diego, Calif, USA, 2008.

[11] M. Baudrit, C. Algora, I. Rey-Stolle, I. García, and B. Galiana, "Numerical analysis of GaInP solar cells: toward advanced photovoltaic devices modeling," in Proceedings of the 5th International Conference on Numerical Simulation of Optoelectronic Devices, pp. 41-42, Berlin, Germany, September 2005.

[12] S. Adachi, Properties of Group-IV, III-V and II-VI Semiconductors, John Wiley \& Sons, Chichester, UK, 2009.

[13] F. Dimroth, U. Schubert, A. W. Bett et al., "Next generation GaInP/GaInAs/Ge multi-junction space solar cells + Fig1, Fig2," in Proceedings of the 17th European Space Power Conference, 2001.

[14] S. P. Philipps, W. Guter, E. Welser et al., "Present status in the development of III-V multi-junction solar cells," in Next Generation of Photovoltaics, vol. 165 of Springer Series in Optical Sciences Volume, pp. 1-21, 2012.

[15] Z. Ouennoughi and M. Chegaar, "Simpler method for extracting solar cell parameters using the conductance method," SolidState Electronics, vol. 43, no. 11, pp. 1985-1988, 1999.

[16] M. Chegaar, Z. Ouennoughi, F. Guechi, and H. Langueur, "Determination of solar cells parameters under illuminated conditions," Journal of Electron Devices, vol. 2, pp. 17-21, 2003.

[17] Z. Q. Li and S. Li, "Sophisticated models replicate the effects of tunnel junctions," Compound Semiconductor, vol. 13, no. 6, pp. 29-31, 2007.

[18] J. F. Wheeldon, C. E. Valdivia, A. Walker et al., "AlGaAs tunnel junction for high efficiency multi-junction solar cells: simulation and measurement of temperature-dependent operation," in Proceedings of the 34th IEEE Photovoltaic Specialists Conference (PVSC '09), pp. 000106-000111, June 2009. 

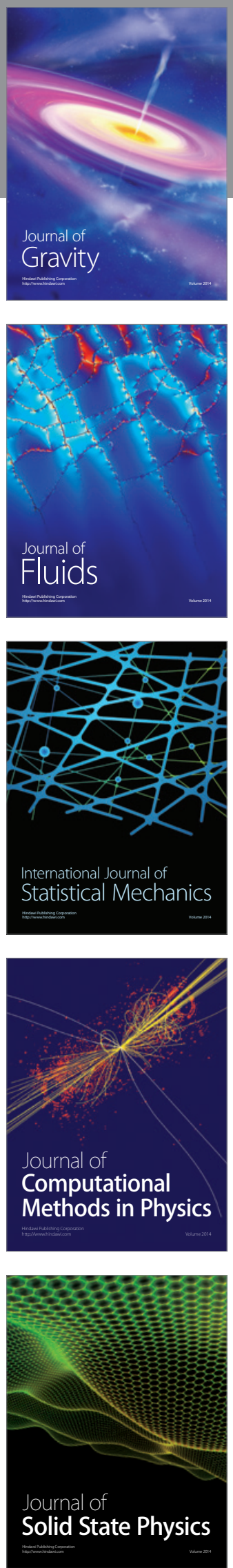

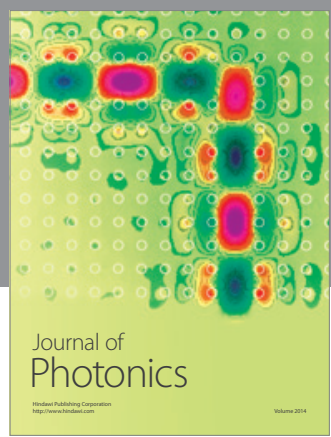

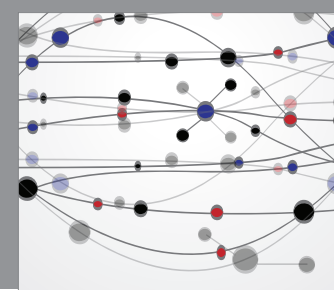

The Scientific World Journal

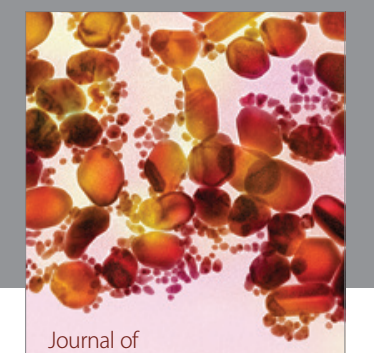

Soft Matter
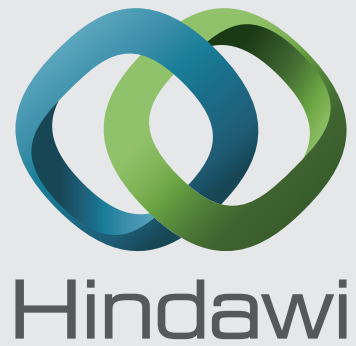

Submit your manuscripts at

http://www.hindawi.com
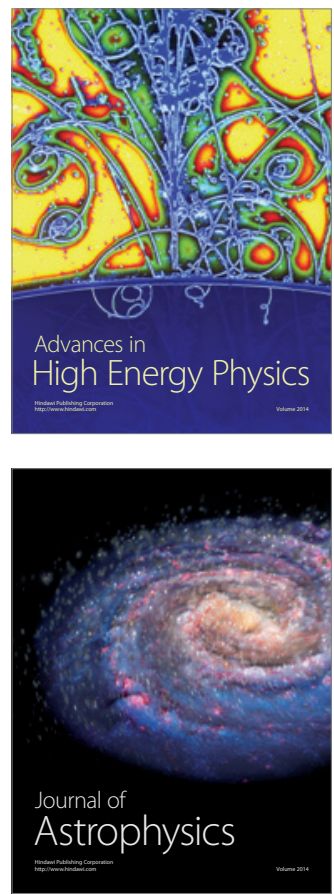
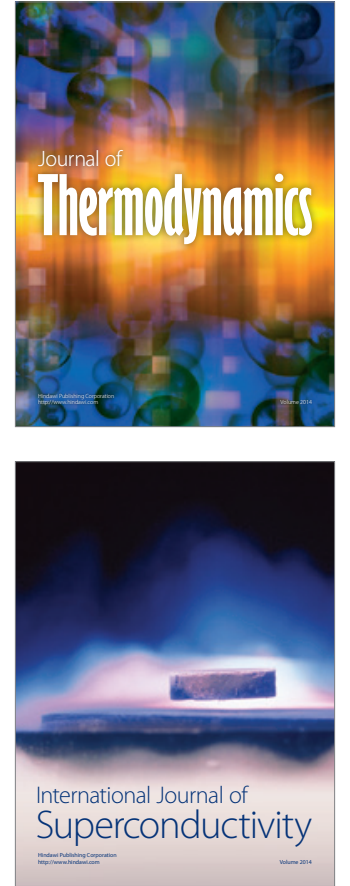
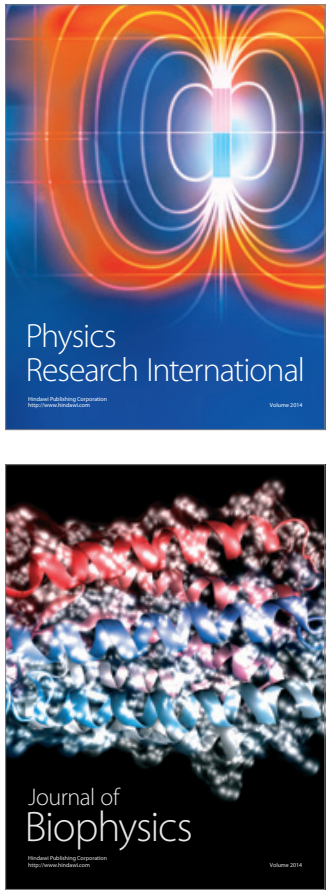
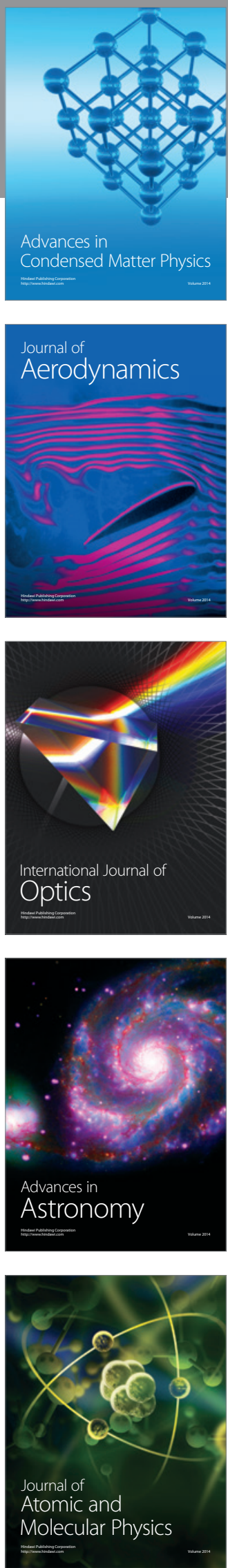